INPLASY PROTOCOL

To cite: Ching et al. Efficacy and safety of scalp acupuncture on hemiplegia after stroke: a protocol for a Meta-analysis and systematic review of randomized controlled trial. Inplasy protocol 202120044. doi: 10.37766/inplasy2021.2.0044

Received: 13 February 2021

Published: 13 February 2021

Corresponding author: Yuen Lim Ching

xmastar1225@hotmail.com

Author Affiliation:

Beijing University of Chinese Medicine

Support: None.

Review Stage at time of this submission: Preliminary

searches.

Conflicts of interest: None declared.

\section{Efficacy and safety of scalp acupuncture on hemiplegia after stroke: a protocol for a Meta-analysis and systematic review of randomized controlled trial}

\author{
Ching, YL1; Liu, ZD²; Zou, Y33; Li, QQ4; Li, YQ5; Li, WX6.
}

Review question / Objective: Some question including: Whether the advantages of scalp acupuncture in clinical trials are superior to other acupuncture methods or conventional massage techniques and conventional rehabilitation treatment methods; Whether researchers in related fields can meet the standards or are subject to restrictions when conducting randomized controlled trials, such as blinding and sample size; Key issues such as the effectiveness and safety of scalp acupuncture in the treatment of hemiplegia after stroke have not yet been comprehensively evaluated. This study aims to systematically evaluate the efficacy and safety of scalp acupuncture in improving hemiplegia in patients with stroke.

Condition being studied: Nowadays, scalp acupuncture is very effective for improving hemiplegia in stroke patients. However, the efficacy and safety of scalp acupuncture to improve the quality of life of hemiplegia after stroke is better than other therapies, there is still a lack of evidence for systematic reviews and meta-analysis.

INPLASY registration number: This protocol was registered with the International Platform of Registered Systematic Review and Meta-Analysis Protocols (INPLASY) on 13 February 2021 and was last updated on 13 February 2021 (registration number INPLASY202120044).

\section{INTRODUCTION}

Review question / Objective: Some question including: Whether the advantages of scalp acupuncture in clinical trials are superior to other acupuncture methods or conventional massage techniques and conventional rehabilitation treatment methods; Whether researchers in related fields can meet the standards or are subject to restrictions when conducting randomized controlled trials, such as blinding and sample size; Key issues such as the effectiveness and safety of scalp acupuncture in the treatment of hemiplegia after stroke have not yet been 
comprehensively evaluated. This study aims to systematically evaluate the efficacy and safety of scalp acupuncture in improving hemiplegia in patients with stroke.

Condition being studied: Nowadays, scalp acupuncture is very effective for improving hemiplegia in stroke patients. However, the efficacy and safety of scalp acupuncture to improve the quality of life of hemiplegia after stroke is better than other therapies, there is still a lack of evidence for systematic reviews and meta-analysis.

\section{METHODS}

Search strategy: (1) Stroke OR apoplexia OR apoplexy OR wind-stroke syndrome OR paralytic stroke OR cerebrovascular accident OR CVA OR cerebrovascular apoplexy OR cerebral vascular disease OR CVD OR cerebral infarction OR cerebral hemorrhage (2) Hemiparalysis OR hemiparesis OR hemiplegia OR hemiplegic OR semisideratio OR semisideration OR paralysis OR paralysation OR plegia OR palsy (3) Scalp acupuncture OR head acupuncture OR scalp needle OR scalp needling OR head needle OR head needling OR Scalp stimulation (4) Randomized controlled trial OR randomized OR placebo (5) (1) And (2) And (3) And (4).

Participant or population: There are clear diagnostic criteria for disease names in Chinese medicine and or Western medicine. Such as ischemic stroke, cerebral infarction, cerebral infarction, cerebral thrombosis, ischemic stroke, ischemic cerebrovascular disease, and ischemic stroke. Patients with hemiplegia after stroke, who have symptoms of restricted limb movement, have a clear diagnosis of the cause, and are clinically diagnosed with stroke. The patient's age, gender, race, etc. are not limited.

Intervention: Mainly for scalp acupuncture that takes acupuncture points on the head zone line.
Comparator: Conventional medication, rehabilitation training, body acupuncture or other measures, etc., are not limited in type.

Study designs to be included: Registered or published randomized controlled trials focusing on scalp acupuncture treatment of hemiplegia after stroke.

Eligibility criteria: Original studies of randomized controlled trials (RCTs) of the treatment of scalp acupuncture in improving hemiplegia after stroke will be included without publication status restriction or writing language.

Information sources: Clinical Trials Center, PubMed, Embase, Cochrane Library, the Chongqing VIP Chinese Science and Technology Periodical Database, Chinese Biological and Medical database, China National Knowledge Infrastructure, and Wanfang database.

Main outcome(s): Fugl-Meyer assessment.

Additional outcome(s): Barthel index score (including the modified Barthel index), Neurological Deficit Score (including NHI stroke scale and Chinese version of $\mathrm{NIH}$ stroke scale), and occurrence of adverse events. 2.4. Exclusion.

Data management: Note express software will be used for literature management. All relevant articles of full text that meet these criteria will be obtained. In case of different opinions, a third reviewer will make the final decision. Two reviewers will separately complete the extraction. Under the instruction of the Cochrane Collaboration, Excel 2019 is used to set up a data extraction table to extract data, including study identification (title, authors, journal, publication year, and country), participants information (age, sample size, sex ratio, course of disease), randomization method, concealment, interventions in treatment and control groups, outcomes, adverse events, and other details. Divergence will be solved by consulting and discussing with the third author. 
Quality assessment / Risk of bias analysis: Two researchers independently will evaluate the risk of bias in RCTs in accordance with the Cochrane Handbook of Systematic Reviewers, including the following items: random sequence generation, allocation concealment, blinding of participants and personnel, blinding of outcome assessment, incomplete outcome data, selective reporting, and other bias. The quality of studies is classified as being at of high, unclear or low risk of bias. In case of disagreement, a third researcher will decide.

Strategy of data synthesis: The RevMan 5.4.1 software provided by the Cochrane Collaboration will be used for statistical analysis. Odd Risk (OR) is selected as the statistic for the dichotomous variable. For continuous variables, weighted mean difference is selected when the tools and units of measurement indicators are the same, standardized mean difference is selected with different tools or units of measurement, and all the above are represented by effect value and $95 \%$ confidence interval. Heterogeneity test: $\mathbf{Q}$ test is used to qualitatively determine interstudy heterogeneity. If $\mathrm{P} \geq .1$, there is no inter-study heterogeneity; If $\mathrm{P} 50 \%$, it is considered to be significant heterogeneity, the source of heterogeneity will be explored through subgroup analysis or sensitivity analysis. If there is no obvious clinical or methodological heterogeneity, it will be considered as statistical heterogeneity, and the random-effect model will be used for analysis. Descriptive analysis will be used if there is significant clinical heterogeneity between the 2 groups and subgroup analysis is not available.

Subgroup analysis: Subgroup analysis is conducted according to the course of disease, which will be divided into acute state, recovery state, and sequelae stage.

Sensitivity analysis: In order to test the stability of meta analysis results of indicators, a one-by-one elimination method will be adopted for sensitivity analysis.
Language: There are no language limits.

Country(ies) involved: China and Malaysia.

Keywords: scalp acupuncture; hemiplegia after stroke; Meta-analysis; systematic review.

Contributions of each author:

Author 1 - Yuen Lim Ching.

Author 2 - Zhidan Liu.

Author 3 - Yuan Zou.

Author 4 - Qiaoqiao Li.

Author 5 - Yingqiu Li. 